ORIGINAL ARTICLE

\title{
An in vitro model for the growth and analysis of chronic wound MRSA biofilms
}

\author{
A.M. Agostinho, A. Hartman, C. Lipp, A.E. Parker, P.S. Stewart and G.A. James \\ Center for Biofilm Engineering, Montana State University, Bozeman, MT, USA
}

\section{Keywords}

antimicrobial agents, biofilms, chronic wounds, in vitro model, MRSA.

\section{Correspondence}

Alessandra M. Agostinho, Center for Biofilm Engineering, 366 EPS Building, Montana State University, Bozeman, MT, USA.

E-mail: aagostinho@erc.montana.edu

2011/0613: received 11 April 2011, revised 16 June 2011 and accepted 21 August 2011

doi:10.1111/j.1365-2672.2011.05138.x

\begin{abstract}
Aims: To develop an in vitro model (Colony/drip-flow reactor - C/DFR) for the growth and analysis of methicillin-resistant Staphylococcus aureus (MRSA) biofilms.

Methods and Results: Using the C/DFR model, biofilms were grown on the top of polycarbonate filter membranes inoculated with a clinical isolate of MRSA, placed on absorbent pads in the DFR and harvested after $72 \mathrm{~h}$. The biofilms varied from 256 to $308 \mu \mathrm{m}$ in thickness with a repeatability standard deviation of $0 \cdot 22$. Testing of antimicrobial agents was also performed where C/DFR biofilms were grown in parallel with conventional colony biofilms. A saline solution (control), 1\% silver sulfadiazine solution, and $0 \cdot 25 \%$ Dakin's solution were used to treat the biofilms for $15 \mathrm{~min}$. Microscopic evaluation of biofilm morphology and thickness was conducted. The Dakins solution in both models produced statistically significantly higher log reductions than silver sulfadiazine treatment.

Conclusions: The C/DFR biofilms were thick and repeatable and exhibited higher resistance to Dakins solution than the treated colony biofilms.

Significance and Impact of the Study: The C/DFR can be used as a tool for examining complex biofilm physiology as well as for performing comparative experiments that test wound care products and novel antimicrobials.
\end{abstract}

\section{Introduction}

Chronic wounds are an important world public health problem that has increased with the ageing and obesity of the population (Miller et al. 1992).

Nonhealing wounds, whose main forms are venous, pressure and diabetic ulcers, affect approx. $1 \%$ of the US population, resulting in suffering, disability and a large burden on financial resources (Ruckley 1997). More than five billion dollars are spent in the treatment for pressure ulcers every year (Brown and Smith 1999), and in 2005, 4.8 in 1000 Americans with diabetes suffered lowerextremity amputations according to Centers for Disease Control and Prevention despite the effort directed at prevention in the last decade.

Unlike acute wounds, chronic wounds lack an orderly and predictable process of healing (American Diabetes
Association 1999). In normal wound healing, keratinocytes, endothelial cells and fibroblasts are coordinated by a complex group of interactions involving cells and matrix, but in chronic wounds, there is a failure in this process leading to a defective wound matrix and lack of re-epithelization (Herrick et al. 1992; Martin 1997).

According to Davies et al. (2001), although there is no single theory to explain the behaviour of chronic wounds, it is likely that the aetiology is multifactorial, including ischaemia, trauma and infection (Tarnuzzer and Schultz 1996). The presence of infection, in which bacteria release toxins in the wound bed and cause an increased inflammatory response, thrombocytopenia and platelet agglutination, can impair the normal sequence of wound healing (Brown and Smith 1999).

Wounds are susceptible to microbial contamination from both exogenous and endogenous sources, including 
the nose, skin, mouth and gut, and it is likely that such organisms are involved in the evolution of resistant microbial communities (Percival and Bowler 2004).

The control of bioburden is known as an important aspect of wound management as microbial infection has deleterious effects on wound healing, but for decades, the bacteria in chronic infections were considered to be growing in planktonic state (James et al. 2008). This belief has been changing over the past years with the increasing acknowledgement that the bacteria-colonizing chronic wounds are organized in complex communities and enmeshed in an exopolysaccharide matrix as biofilms (Bjarnsholt et al. 2008; James et al. 2008).

The impaired penetration of antibiotics in these communities, the reduced growth rate of bacteria, the altered micro-environment, the particular gene expression and the presence of an intricate cell to cell communication system (quorum sensing) make the biofilm difficult to treat and eliminate (Percival and Bowler 2004). Biofilms tolerance to antibiotics and other antimicrobials can be hundreds to thousands times higher when compared to planktonic bacteria (Ceri et al. 1999) and of further concern is the emergence of the so-called super bugs such as methicillin-resistant Staphylococcus aureus (MRSA), which have been found more frequently infecting chronic wounds (Demling and Waterhouse 2007).

As stated by Percival and Bowler (2004), wound biofilm research is necessary to improve the understanding of the impact of biofilms and bacterial interactions on wound healing and this is the aim of the Colony/dripflow reactor $(\mathrm{C} / \mathrm{DFR})$ in vitro model described in this manuscript.

\section{Materials and methods}

\section{Model development}

The model described in this study is a combination of the colony biofilm model (Anderl et al. 2000) and the DFR (Xu et al. 1998). Photographs of the model are shown in Fig. 1. A six-chamber DFR (Biosurface Tech- nologies Corporation, Bozeman, MT, USA) was assembled using plain glass slides, to which 25-mm Millipore absorbent pads were attached with silicone aquarium sealant. This apparatus was sterilized by autoclaving for 25 min. Black polycarbonate filter membranes with 0.22$\mu \mathrm{m}$ pore size and $25 \mathrm{~mm}$ in diameter (Osmonics, Inc., Minnetonka, MN, USA) were sterilized with germicidal UV light for 15 min on each side and positioned over the absorbent pads in the DFR. A MRSA clinical wound isolate obtained from the South West Regional Wound Care Clinic in Lubbock, Texas, was used as inoculum. Absorbance of the overnight culture was measured at a 600-nm wavelength, using a spectrophotometer, and the culture was diluted with sterile tryptic soy broth (TSB) to obtain a 0.05 absorbance. The average log colony-forming units per $\mathrm{ml}\left(\mathrm{CFU} \mathrm{ml}{ }^{-1}\right)$ in the inocula was $4 \cdot 26 \pm 0 \cdot 44$. The membranes were inoculated with $10 \mu$ lof the diluted overnight culture of MRSA and allowed to dry for 30 min earlier starting the medium flow. The reactor was operated at room temperature using a peristaltic pump to provide a flow rate of $5 \mathrm{ml} \mathrm{h}^{-1}$ of $10 \%$-strength TSB to each channel. After $72 \mathrm{~h}$, the polycarbonate membranes were transferred to $10 \mathrm{ml}$ of phosphate buffer solution and the biofilms were dispersed by a vortexing/sonication procedure, serially diluted and plated on tryptic soy agar (TSA) in triplicate. Following $24 \mathrm{~h}$ of incubation at $37^{\circ} \mathrm{C}$, the colonies on the plates were counted and the colony-forming units per membrane $\left(\mathrm{CFU} \mathrm{M} \mathrm{M}^{-1}\right.$ ) were calculated. This experiment was conducted five times to evaluate the within-experiment and among-experiment variability of the C/DFR model. The CFU M ${ }^{-1}$ were log-transformed and analysed using a random effects analysis of variance (ANOVA) which was fit using the computer software package MinitaB (Release 13; Minitab, State College, PA, USA). The ANOVA provided estimates of the variance because of within-experiment sources and the variance because of among-experiment sources (Pinheiro and Bates 2000). The square root of the sum of those two variances is the repeatability standard deviation, which is interpreted as the typical distance between the log density for a single
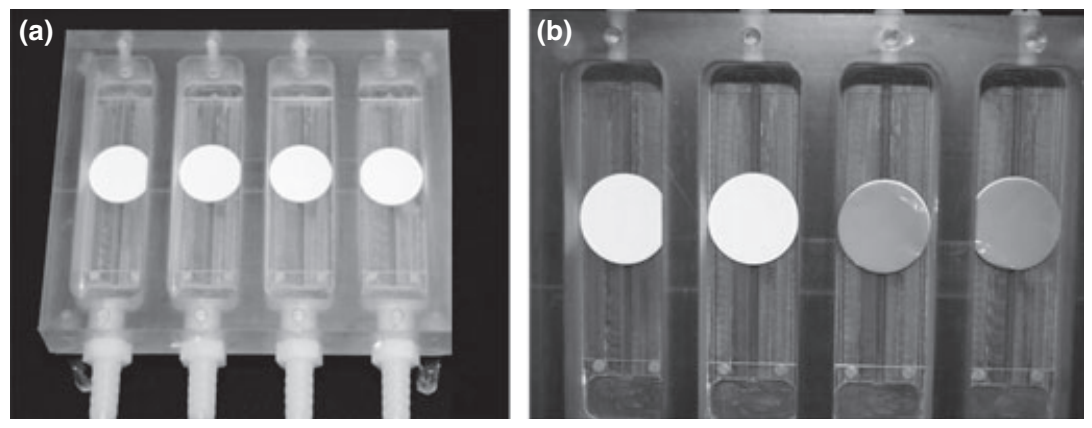

Figure 1 C/DFR setup (a) general view; (b) close-up view of the biofilm. C/DFR, Colony/drip-flow reactor. 
(randomly chosen) experiment and the mean log density across many independent, identical (same operational factor settings in the same laboratory) experiments.

Testing of antimicrobial agents and comparison with the colony biofilm model

Another series of experiments was conducted to evaluate the efficacy of common antimicrobial agents in reducing/killing MRSA biofilms grown in the C/DFR. The same agents were applied side-by-side to biofilms grown in the colony model, and the log reductions in the two models were compared. The C/DFR was prepared as described earlier, and the membranes were inoculated with $10 \mu \mathrm{l}$ of the MRSA wound clinical isolate. The biofilms were grown at room temperature with a flow rate of $5 \mathrm{ml} \mathrm{h}^{-1}$ per chamber of $10 \%$-strength TSB.

The colony biofilms were grown on black UV-sterilized polycarbonate membrane filters (diameter $25 \mathrm{~mm}$, pore size $0.22 \mu \mathrm{m}$; Osmonics, Inc.) resting on $10 \%$-strength TSA plates. An overnight culture of the MRSA clinical isolate was diluted to an optical density of 0.05 at $600 \mathrm{~nm}$ in TSB, and a $10 \mu \mathrm{l}$ drop was used to inoculate each membrane.

Upon drying of the inoculum, the plates were inverted and incubated at $37^{\circ} \mathrm{C}$ for $72 \mathrm{~h}$, with the membrane-supported biofilm transferred to fresh culture medium after every $24 \mathrm{~h}$.

After $72 \mathrm{~h}$, the biofilms on two of the six C/DFR slides and the corresponding colony biofilms were each treated with saline solution (control), 1\% silver sulfadiazine solution $\left(1000 \mu \mathrm{g} \mathrm{ml}^{-1}\right)$, or a $0 \cdot 25 \%$ Dakin's solution. The treatments were applied for $15 \mathrm{~min}$ by pipetting $0.5 \mathrm{ml}$ of the treatment solution on top of the biofilms at room temperature. After $15 \mathrm{~min}$, the membranes/biofilms were transferred to $10 \mathrm{ml}$ of Difco ${ }^{\mathrm{TM}}$ Dey Engley neutralizing broth (Fisher Scientific, Pittsburgh, PA, USA) for neutralization, dispersed, serially diluted and plated on TSA. Following $24 \mathrm{~h}$ of incubation at $37^{\circ} \mathrm{C}$, colonies were counted and the $\mathrm{CFU} \mathrm{M}{ }^{-1}$ were calculated. The experiment was repeated four times, and the log reductions relative to saline and the repeatability standard deviation were calculated for each treatment.

The mean log reductions were then compared using Welch $t$-tests, which accounted for the different variability of each of the treatments.

\section{Microscopic examination}

C/DFR biofilms and colony biofilms were also examined by microscopy for the comparison of architecture and measurement of thickness. Biofilms were grown for 3 days as described earlier, embedded in OCT (Optimum Cutting Temperature; Sakura Finetek, Torrance, CA, USA), frozen and cryosectioned in $5-\mu$ m-thickness sections using a cryostat.

The sections were examined using light microscopy with a Nikon Eclipse E800 microscope with a 100× oil immersion objective (Nikon, Melville, NY, USA). Images were taken with a Photometrics CoolSNAP EZ camera (Photometrics, Tucson, AZ, USA) and analysed with MetaMorph 7.5 software (Molecular Devices, Sunnyvale, CA, USA).

\section{Results}

MRSA biofilms grown in the C/DFR over five independent experiments grew roughly within the confines of the inoculated area of the membranes to form circular biofilms with a diameter of approx. $5 \mathrm{~mm}$ (Fig. 2a,b). The density of bacteria within these biofilms ranged from a $\log$ CFU $M^{-1}$ of $8 \cdot 62-9 \cdot 39$ with a mean $9 \cdot 11$ (Fig. 3 ). The repeatability standard deviation was $0 \cdot 22$, with $64 \%$ of the variability of the data attributable within-experiment sources and $36 \%$ attributable to among-experiment sources.

The log densities from four independent experiments testing the effect of the antimicrobials on the C/DFR biofilms are shown in Fig. 4. The statistical results, given in Table 1, showed an average bacterial density of
Figure 2 MRSA biofilm grown in the C/DFR. (a) General view; (b) close-up view of the biofilm. MRSA, methicillin-resistant Staphylococcus aureus; C/DFR, Colony/drip-flow reactor.
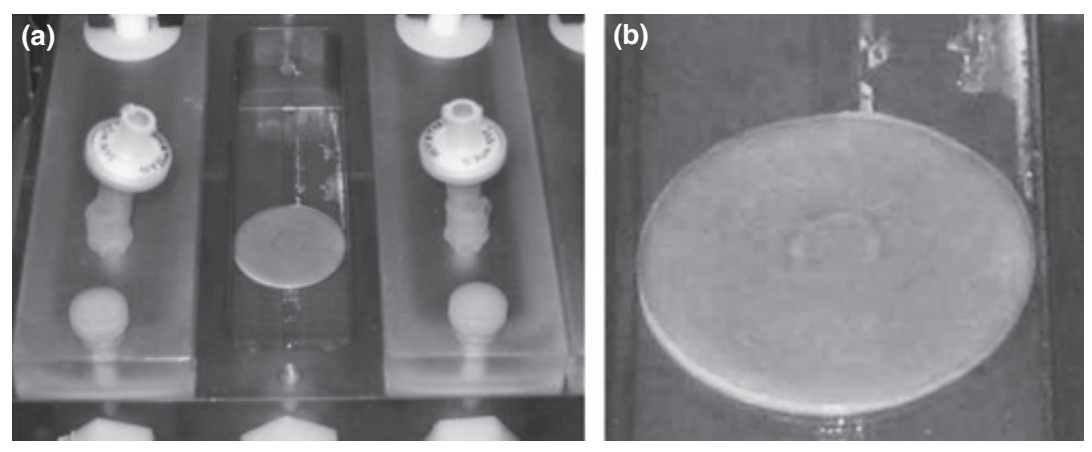

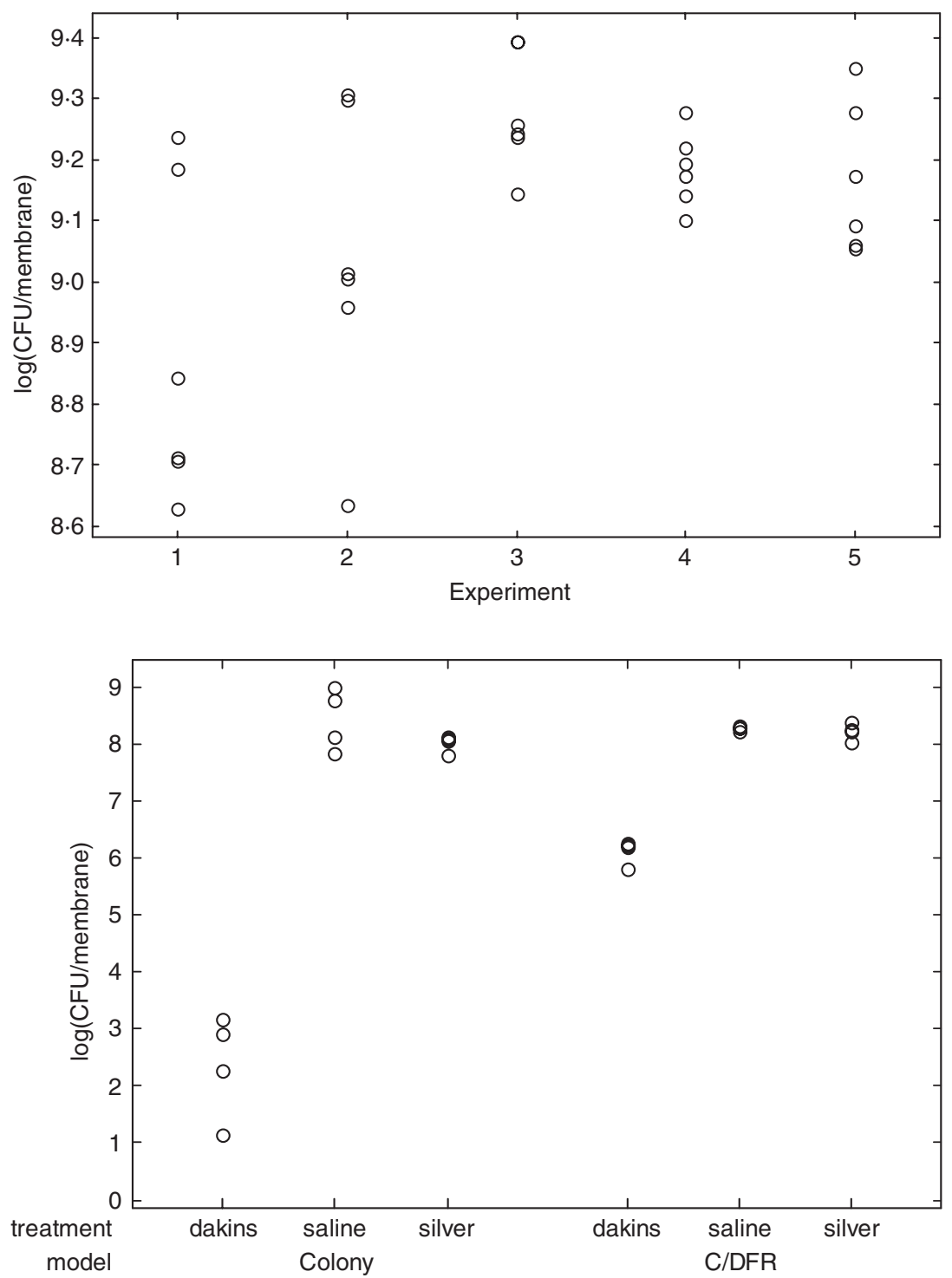

Figure 3 Biofilm log densities from five independent experimental runs of the C/DFR model. C/DFR, Colony/drip-flow reactor.

Figure 4 Biofilm log densities from four independent experimental runs of the C/DFR and colony models run in parallel. C/DFR, Colony/drip-flow reactor.

Table 1 Results of the statistical analysis applied to the four independent experimental runs of the Colony/drip-flow reactor (C/DFR) and colony models run in parallel

\begin{tabular}{|c|c|c|c|c|}
\hline \multirow[b]{2}{*}{ Variable } & \multicolumn{2}{|l|}{ C/DFR } & \multicolumn{2}{|l|}{ Colony } \\
\hline & Mean & $\begin{array}{l}\text { Repeatability } \\
\text { SD }\end{array}$ & Mean & $\begin{array}{l}\text { Repeatability } \\
\text { SD }\end{array}$ \\
\hline LD (saline) & $9 \cdot 274$ & 0.0345 & $9 \cdot 435$ & 0.546 \\
\hline LR (Dakins) & $2 \cdot 157$ & $0 \cdot 1814$ & 6.076 & $1 \cdot 419$ \\
\hline LR (silver) & 0.061 & $0 \cdot 1344$ & 0.422 & 0.431 \\
\hline
\end{tabular}

LD, log density (in CFU per membrane); LR, log reduction; SD, standard deviation.

9.27 $\log$ CFU $\mathrm{M}^{-1}$ for the saline-treated biofilms. The silver sulfadiazine treatment did not present a statistically significant $\log$ reduction in the biofilms $(P$-value $=$
0.431), while the Dakin's solution log reduction was statistically significantly smaller than the saline control $(P$-value $<0.0001)$.

For the colony biofilms, the cell density ranged from a $\log$ CFU $M^{-1}$ of $8 \cdot 83-10 \cdot 00$. An average log density of $9 \cdot 44$ was observed with saline solution treatment. Silver sulfadiazine treatment resulted in a $0.42 \mathrm{log}$ reduction while Dakin's solution reduced the biofilms by $6.08 \mathrm{log}$, a statistically significant difference $(P$-value $=0 \cdot 0030)$ from the control.

When comparing the log reductions for both models, the treatment with silver sulfadiazine did not result in statistically significant differences $(P$-value $=0.9021)$. Dakin's solution, however, was more effective in killing colony biofilms than C/DFR biofilms $(P$-value $=0 \cdot 0001)$. Also, for every treatment including the controls, the 
C/DFR biofilms were more repeatable (see the standard deviations in Table 1).

The microscopic analysis of the biofilms showed that those grown in the C/DFR ranged from 256 to $308 \mu \mathrm{m}$ in thickness and channels between cell clusters were readily apparent (Fig. 5a). However, colony biofilms were approx. $1 / 3$ as thick, ranging from 109 to $119 \mu \mathrm{m}$, and channels were not present as in the C/DFR biofilms (Fig. 5b).

\section{Discussion}

Several methods of biofilm growth have been developed over the years using numerous types of micro-organisms for different applications. Ideally, an in vitro model should mimic the clinical situation as closely as possible (Percival et al. 2007). However, methods like the Colony Model (Anderl et al. 2000), the DFR (Xu et al. 1998), the Capillary Flow Cell (Mittelman et al. 1992) or the CDC reactor (Donlan et al. 2002), while extensively used in our laboratory and capable of producing consistent and repeatable biofilms, fail in our opinion to recreate the unique environment present in wounds.

A method is considered repeatable if independent repeats of the same experiment in the same laboratory produce similar results. The conventional measure of repeatability is the standard deviation (Zelver et al. 1999; Feldsine et al. 2002). The statistical analysis of the data indicated that biofilms grown under both control and active treatment conditions in the C/DFR exhibited excellent repeatability, much better than the colony model from which it was derived. Growth of the biofilms was conducted at a different temperature for each model. We do not believe this difference impacted the repeatability of the models. The repeatability standard deviations for the C/DFR model are also similar to those reported in 2007 by Buckingham-Meyer et al. (2007) for control and treated Staph. aureus biofilms grown in the drip-flow and the CDC reactors, which are approved ASTM International methods. The 'weaker' treatments (defined by the authors as treatments that produced a $\log$ reduction $\leq 1 \cdot 0$ ) were comparable to silver sulfadiazine in our study, and 'stronger' treatments (that produced a log reduction $>1 \cdot 0$ ) were comparable to Dakins solution in our study (Table 2).

Recently, several manuscripts describing new in vitro models for the growth of chronic wound biofilms have been published (Sun et al. 2008; Hill et al. 2010; Kostenko et al. 2010 and Werthen et al. 2010), and although they contribute to the scientific and clinical research of wound biofilms, they fail to mimic the clinical environment of wounds. The in vitro multispecies model specific for reproducing chronic wound biofilms developed by Sun et al. (2008) allows the growth of biofilms in $24 \mathrm{~h}$ for rapid screening of therapeutics; however, such a model produces microbial communities fully immersed in liquid nutrient medium, a situation that dramatically differs

Figure 5 Micrographs of biofilms. (a) C/DFR MRSA biofilm; (b) colony MRSA biofilm. MRSA, methicillin-resistant Staphylococcus aureus; C/DFR, Colony/drip-flow reactor.
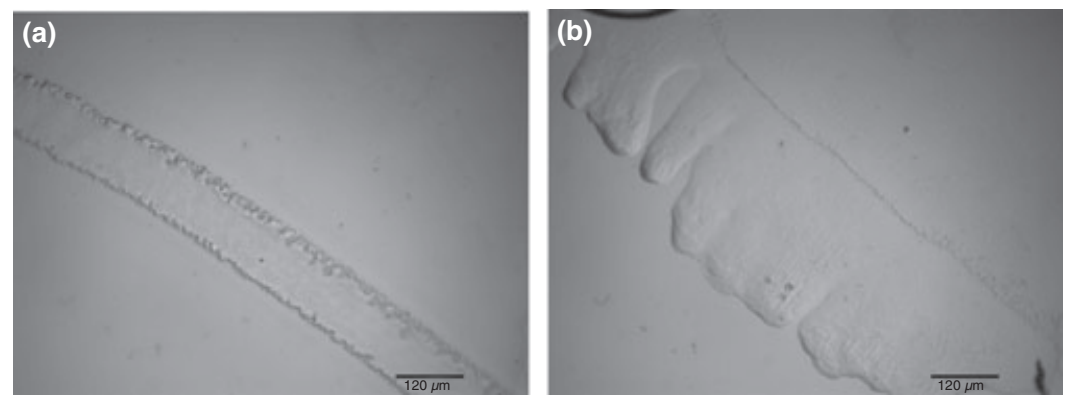

Table 2 Comparison of the results of the Colony/drip-flow reactor (C/DFR) and colony models for Staphylococcus aureus from this paper (first two rows of the table) to the results for the CDC reactor and DFRs (3rd-4th rows of the table) reported in Buckingham-Meyer et al. (2007)

\begin{tabular}{|c|c|c|c|c|c|c|}
\hline \multirow{2}{*}{\multicolumn{3}{|c|}{ Control biofilms }} & \multicolumn{4}{|c|}{ Treated biofilms } \\
\hline & & & \multicolumn{2}{|c|}{ Weaker treatment } & \multicolumn{2}{|c|}{ Stronger treatment } \\
\hline Model & Mean LD & Repeatability SD & Mean LR & Repeatability SD & Mean LR & Repeatability SD \\
\hline C/DFR & $9 \cdot 1$ & 0.223 & 0.061 & $0 \cdot 13$ & $2 \cdot 157$ & $0 \cdot 1814$ \\
\hline Colony & $9 \cdot 4$ & 0.546 & 0.422 & 0.431 & $6 \cdot 08$ & $1 \cdot 419$ \\
\hline DF & $8 \cdot 6$ & 0.586 & $<1$ & $1 \cdot 34$ & $>1$ & $1 \cdot 27$ \\
\hline CDC & $8 \cdot 3$ & 0.224 & $<1$ & $0 \cdot 31$ & $>1$ & $0 \cdot 36$ \\
\hline
\end{tabular}

$L D$, log density; LR, log reduction; SD, standard deviation. 
from the clinical reality. The same problem is observed in the models presented by Hill et al. (2010) using a constant depth film fermenter and Kostenko et al. (2010) that used MBEC plates (MBEC ${ }^{\text {TM }}$ high-throughput (HTP) assay plates) to grow wound biofilms. Werthen et al. (2010) published a very interesting in vitro model for the growth of chronic wounds and other soft-tissue biofilms using collagen matrices, but for the entire period of biofilm growth, there is no addition of fresh medium simulating the continuous production of exudate by the wound. The method described here was developed with the objective of reproducing some characteristics of the environment of the wound, in which the exudate perfuses to the surface of the ulcer, feeding the biofilm that stays in contact with air. In addition, the flow rate of the medium through the reactor can be adjusted to reflect the exudate production observed in different types of chronic wounds, and the temperature for biofilm growth can be altered to reflect contrasts in clinical conditions present in different limb diseases.

As the biofilms grown in the C/DFR are supported by polycarbonate membranes, it enables the transfer of the biofilms from the reactor for various types of analysis, such as confocal microscopy, scanning electron microscopy, fluorescence microscopy and light microscopy following cryosectioning, all without disturbance of the morphology or structure of the biofilms.

Considering that a chronic wound that presents $>10^{5}$ bacteria per gram of tissue has been considered 'infected' (Robson 1997), the biofilms grown in the C/DFR over the period of 3 days of incubation, which exhibited about $10^{9}$ bacteria per membrane, may be representative of those in severely infected wounds. Even though this level of bioburden is unlikely under the care of a competent wound care professional, it does provide advantages for understanding the dynamics of growth of chronic wound biofilms as well as provides a more realistic test of antimicrobials when compared to testing against planktonic bacteria.

In the last three of five experiments with the C/DFR, the variability in $\log \mathrm{CFU} \mathrm{M}{ }^{-1}$ was lower than the first two experiments. This may have been due to the technician gaining more experience with the technique.

Increasing evidence suggests that micro-organisms inhabiting chronic wounds exist as biofilms, but traditional research, development and testing of antimicrobial agents have focused on the more-susceptible planktonic bacteria (Costerton et al. 1995, 2003). Furthermore, important emerging multi-drug resistant strains such as vancomycinintermediate Staph. aureus, vancomycin-resistant Enterococcus and MRSA have not been thoroughly studied.

Even though there is no single answer to why bacterial cells living as biofilms present increased antimicrobial tol- erance, several mechanisms seem to be involved: penetration of antibiotics, physiological state of the bacteria, heterogeneity within the biofilm, general stress response, quorum sensing and the altered phenotype (Mah and O'Toole 2001).

While the colony biofilms replicate some of the chronic wound environment characteristics by allowing the growth of biofilms on a semi-solid medium in contact with air, the microscopic comparison of the C/DFR and colony biofilms justifies the use of the former based on the differences between biofilm thickness and morphology. Although the C/DFR biofilms were grown at room temperature and the colony biofilms at $37^{\circ} \mathrm{C}$, the bacterial densities on the membranes for both methods were around the same order of magnitude $\left(10^{9} \mathrm{CFU} \mathrm{M}^{-1}\right)$, the thickness and the morphology of the biofilms differed dramatically. Several studies have shown that the penetration of an antimicrobial can be influenced by the bacterial composition of the biofilm as well as the type of antimicrobial applied (Mah and O'Toole 2001) but a common observation is that independently of these variables, the thicker a biofilm is, the more tolerant it seems to be. The increased thickness of the C/DFR biofilms (on average, triple the thickness of the colony biofilms), although grown at room temperature implies that a greater amount of extracellular polymeric matrix is present, which may play an important role in the high tolerance to antimicrobials observed in this model. We predict that colony biofilms grown at room temperature would be thinner and have lower cell density than those grown at $37^{\circ} \mathrm{C}$ in this study. Biofilms grown at room temperature would be less comparable to the C/DFR biofilms, which have similar cell densities to the $37^{\circ} \mathrm{C}$ colony biofilms. Furthermore, we believe that the selection of an in vitro biofilm model that produces a more complex biofilm morphology like the C/DFR over the colony biofilms represents a more stringent challenge to the efficacy of antimicrobials.

De Beer et al. (1994) used selective chlorine microeletrodes to measure chlorine penetration within a mixed species biofilm of Pseudomonas aeruginosa and Klebsiella pneumoniae and verified that the limited penetration is not due simply to transient diffusion but is caused by the neutralization of the chlorine in the biofilm matrix, characterized as a reaction-diffusion interaction. Similarly, the low efficacy of the Dakin's solution against the C/DFR biofilms may be due to chlorine inactivation by the matrix and is also evidence that supports the suggestion that this model is a good in vitro representation of recalcitrant clinical biofilms.

In spite of the fact that the antimicrobial properties of silver have been recognized for a long time and its broad spectrum against bacteria including MRSA has been proclaimed, the testing of the $1 \%$ silver sulfadiazine solution 
(1000 $\mu \mathrm{g} \mathrm{ml}^{-1}$ ) had little, if no effect against the biofilms grown in this model. Bjarnsholt et al., in 2007, while treating Ps. aeruginosa biofilms grown in continuous-culture once-through flow chambers verified that concentrations of silver sulfadiazine between 5 and $10 \mu \mathrm{g} \mathrm{ml}^{-1}$ were able to eradicate the biofilms in $24 \mathrm{~h}$, while a lower concentration of $1 \mu \mathrm{g} \mathrm{ml}^{-1}$ had no effect. The discrepancy in the effect of silver sulfadiazine in the study performed by Bjarnsholt et al. (2007) and our study, that used a much higher concentration of silver sulfadiazine, may be due to the shorter duration of our treatment but could also indicate that the C/DFR model produces biofilms that are more tolerant to antimicrobial agents.

Wounds can support a very diverse and complex microflora even including strict anaerobes (Dowd et al. 2008) and it is likely that the interactions between these different micro-organisms have a synergistic effect, increasing their pathogenicity. Trengove et al. (1996) found that wounds with four or more bacterial species had a significantly lower rate of healing when compared to wounds infected with fewer species of bacteria. Thus, a system that facilitates the study of the interactions between micro-organisms is highly desirable. The C/DFR model is not restricted to the development of single species communities; it allows the introduction of multiple species in the biofilm, including anaerobes, as well as the use of selective or reinforced growth media. Another advantage of this model is that the treatments can be applied on the top of the biofilms simulating a topical treatment; pumped through the system, mimicking a systemic treatment; or used both topically and systemically to evaluate the performance of a dual approach.

Although it is recognized that in vitro data cannot be directly extrapolated to clinical outcomes (Percival et al. 2007) and that more sophisticated models such as biofilm/cell culture, biofilm/tissue culture and a variety of animal models are being developed to investigate biofilm-host interactions, the value of in vitro models cannot be overlooked. In vitro models allow the study of complex phenomena that are not observed using traditional culture methods and that may be difficult to evaluate in vivo. Furthermore, in vitro models can be used to test antimicrobial agents that may facilitate wound management by providing a screening mechanism prior to further testing in in vivo models and clinical trials.

In conclusion, the C/DFR model is a repeatable, relatively simple and a particularly versatile in vitro system for growing several types of biofilms including multi-species that can be used as a tool for understanding complex biofilm physiology as well as for performing comparative experiments testing wound care products and novel antimicrobials.

\section{Acknowledgements}

The project described was supported by grant number 1P20GM078445-01 from the National Institute of General Medical Sciences (NIGMS). The contents of this project are solely the responsibility of the authors and do not necessarily represent the official views of the NIGMS.

\section{References}

American Diabetes Association (1999) Consensus development conference on diabetic foot wound care. Diabetes Care 22, 1354-1360.

Anderl, J.N., Franklin, M.J. and Stewart, P.S. (2000) Role of antibiotic penetration limitation in Klebsiella pneumoniae biofilm resistance to ampicillin and ciprofloxacin. Antimicrob Agents Chemother 44, 1818-1824.

de Beer, D., Srinivasan, R. and Stewart, P.S. (1994) Direct measurement of chlorine penetration into biofilms during disinfection. Appl Environ Microbiol 60, 4339-4344.

Bjarnsholt, T., Kirketerp-Moller, K., Kristiansen, S., Phipps, R., Nielsen, A.K., Jensen, P.O., Hoiby, N. and Givskov, M. (2007) Silver against Pseudomonas aeruginosa biofilms. APMIS 115, 921-928.

Bjarnsholt, T., Kirketerp-Moller, K., Jensen, P.O., Madsen, K.G., Phipps, R., Krogfelt, K., Hoiby, N. and Givskov, M. (2008) Why chronic wounds will not heal: a novel hypothesis. Wound Repair Regen 16, 2-10.

Brown, D.L. and Smith, D.J. (1999) Bacterial colonization/infection and the surgical management of pressure ulcers. Ostomy Wound Manage 45, 109S-118S.

Buckingham-Meyer, K., Goeres, D.M. and Hamilton, M.A. (2007) Comparative evaluation of biofilm disinfectant efficacy tests. J Microbiol Methods 70, 236-244.

Centers for Disease Control and Prevention. Diabetes public health resource. Available at: http://www.cdc.gov/diabetes/ statistics/lealevel/fig8.htm (accessed 10 March 2011).

Ceri, H., Olson, M.E., Stremick, C., Read, R.R., Morck, D. and Buret, A. (1999) The Calgary biofilm device: new technology for rapid determination of antibiotic susceptibilities of bacterial biofilms. J Clin Microbiol 37, 1771-1776.

Costerton, J.W., Lewandowski, Z., Caldwell, D.E., Korber, D.R. and Lappin-Scott, H.M. (1995) Microbial biofilms. Annu Rev Microbiol 49, 711-745.

Costerton, J.W., Veeh, R., Shirtliff, M., Pasmore, M., Post, C. and Ehrlich, G. (2003) The application of biofilm science to the study and control of chronic bacterial infections. J Clin Invest 112, 1466-1477.

Davies, C.E., Wilson, M.J., Hill, K.E., Stephens, P., Hill, M.C., Harding, K.G. and Thomas, D.W. (2001) Use of molecular techniques to study microbial diversity in the skin: chronic wounds reevaluated. Wound Repair Regen 9, 32-340.

Demling, R.H. and Waterhouse, B. (2007) The increasing problem of wound bacterial burden and infection in acute 
and chronic soft-tissue wounds caused by methicillin-resistant Staphylococcus aureus. J Burns Wounds 16, 86-98.

Donlan, R.M., Murga, R., Carpenter, J., Brown, E., Besser, R. and Fields, B. (2002) Monochloramine disinfection of biofilm associated Legionella pneumophila in a potable water model system. In Legionella ed. Marre, R. pp. 406-410. Washington, DC: American Society for Microbiology.

Dowd, S.E., Sun, Y., Secor, P.R., Rhoads, D.D., Wolcott, B.M., James, G.A. and Wolcott, R.D. (2008) Survey of bacterial diversity in chronic wounds using pyrosequencing, DGGE, and full ribosome shotgun sequencing. BMC Microbiol 8, 43-58.

Feldsine, P., Abeyta, C. and Andrews, W.H. (2002) AOAC International methods committee guidelines for validation of qualitative and quantitative food microbiological official methods of analysis. J AOAC Int 85, 1187-1200.

Herrick, S.E., Sloan, P., McGurk, M., Freak, L., McCollum, C.N. and Ferguson, M.W. (1992) Sequential changes in histologic pattern and extracellular matrix deposition during the healing of chronic venous ulcers. Am J Pathol 141, 1085-1095.

Hill, K.E., Malic, S., Mckee, R., Rennison, T., Harding, K.G., Williams, D.W. and Thomas, D.W. (2010) An in vitro model of chronic wound biofilms to test wound dressings and assess antimicrobial susceptibilities. J Antimicrob Chemother 65, 1195-1206.

James, G.A., Swogger, E., Wolcott, R., Pulcini, E., Secor, P., Sestrich, J., Costerton, J.W. and Stewart, P.S. (2008) Biofilms in chronic wounds. Wound Repair Regen 16, 37-44.

Kostenko, V., Lyczak, J., Turner, K. and Martinuzzi, R.J. (2010) Impact of silver-containing wound dressings on bacterial biofilm viability and susceptibility to antibiotics during prolonged treatment. Antimicrob Agents Chemother 54, 5120-5131.

Mah, T.C. and O’Toole, G.A. (2001) Mechanisms of biofilm resistance to antimicrobial agents. Trends Microbiol 9, 34-39.

Martin, P. (1997) Wound healing - aiming for perfect skin regeneration. Science 276, 75-81.

Miller, C.C., Miller, M.K., Ghaffari, A. and Kunimoto, B. (1992) Treatment of chronic nonhealing leg ulceration with gaseous nitric oxide: a case study. J Cutan Med Surg 8, 233-238.

Mittelman, M.W., Kohring, L.L. and White, D.C. (1992) Multipurpose laminar-flow adhesion cells for the study of bacterial colonization and biofilm formation. Biofouling $\mathbf{6}$, 39-51.

Percival, S.L. and Bowler, P.G. (2004) Biofilms and their potential role in wound healing. Wounds 16, 234-240.

Percival, S.L., Bowler, P.G. and Dolman, J. (2007) Antimicrobial activity of silver-containing dressings on wound microorganisms using an in vitro biofilm model. Int Wound J 4, 186-191.

Pinheiro, J.C. and Bates, D.M. (2000) Statistics and Computing. New York: Springer.

Robson, M.C. (1997) Wound infection: a failure of wound healing caused by an imbalance of bacteria. Surg Clin North Am 77, 637-650.

Ruckley, C.V. (1997) Socioeconomic impact of chronic venous insufficiency and leg ulcers. Angiology 48, 67-69.

Sun, Y., Dowd, S.E., Smith, E., Rhoads, D.D. and Wolcott, R.D. (2008) In vitro multispecies Lubbock chronic wound biofilm model. Wound Repair Regen 16, 805-831.

Tarnuzzer, R.W. and Schultz, G. (1996) Biochemical analysis of acute and chronic wound environments. Wound Repair Regen 4, 321-325.

Trengove, N.J., Stacey, M.C., McGechie, D.F. and Mata, S. (1996) Qualitative bacteriology and leg ulcer healing. $J$ Wound Care 5, 277-280.

Werthen, M., Henriksson, L., Jensen, P.O., Sternberg, C., Givskov, M. and Bjarnsholt, T. (2010) An in vitro model of bacterial infections in wounds and other soft tissues. APMIS 118, 156-164.

Xu, K.D., Stewart, P.S., Xia, F., Huang, C.T. and McFeters, G.A. (1998) Spatial physiological heterogeneity in Pseudomonas aeruginosa biofilm is determined by oxygen availability. Appl Environ Microbiol 64, 4035-4039.

Zelver, N., Hamilton, M., Pitts, B., Goeres, D., Walker, D., Sturman, P. and Heersink, J. (1999) Measuring antimicrobial effects on biofilm bacteria: from laboratory to field. Methods Enzymol 310, 608-628. 